COMMUNICATION

\section{Thermosensitive dendrimer formulation for drug delivery at physiologically relevant temperatures $\dagger$}

\author{
Annie Castonguay, ${ }^{a}$ Eleanor Wilson, ${ }^{a}$ Noura Al-Hajaj, ${ }^{b}$ Laurène Petitjean, ${ }^{a}$ Julie Paoletti, ${ }^{a}$ \\ Dusica Maysinger ${ }^{* b}$ and Ashok Kakkar $* a$
}

Received 28th August 2011, Accepted 23rd September 2011

DOI: $10.1039 / \mathrm{clcc15354d}$

A simple and versatile dendrimer based platform to deliver therapeutic agents at temperatures within the physiological range, is reported. Lipoic acid conjugated at the periphery of the thermosensitive dendrimer formulations undergoes slow and sustained release at $37-42{ }^{\circ} \mathrm{C}$, and rescues the cells from oxidative stress and a pro-inflammatory endotoxic agent.

Modern therapeutic interventions rely on controlled drug delivery to achieve optimal efficacy, using a stimulus that can be tailored under physiological conditions. ${ }^{1}$ Considerable effort has been recently devoted to the development of nanocarriers in which drug release profiles could be modulated using a variety of stimuli including changes in $\mathrm{pH}$, temperature, light $e t c{ }^{2}$ Among these, thermoresponsive drug delivery systems have offered tremendous potential, and constitute a topical area of research. ${ }^{3}$ Dendrimers are monodisperse hyperbranched macromolecules which are increasingly being evaluated for biomedical applications. ${ }^{4}$ In the past few years, a series of efficient reactions commonly referred to as "click" chemistry, notably the $\mathrm{Cu}(\mathrm{I})$-catalyzed alkyne-azide cycloaddition (CuAAC), the Diels-Alder (DA) reaction and thiol-ene coupling, have emerged in the field of macromolecule functionalizations. ${ }^{5}$ In addition, it has recently been demonstrated that dendrimers can also be efficiently constructed in a layer-by-layer fashion, via a sequence of a combination of these click reactions. ${ }^{6}$ An interesting feature of the dendrimers synthesized using the DA cycloaddition is their heat sensitivity, which leads to reversibility of the reaction. Cleavable dendrimers are well known in the literature. ${ }^{7}$ They have attracted interest for biological applications, and the disassembly of most of the systems reported is triggered by hydrolytic, enzymatic or photolytic processes. Interestingly, the reversible nature of

\footnotetext{
${ }^{a}$ Department of Chemistry, McGill University, 801 Sherbrooke St. West, Montreal, Quebec, H3A 2K6, Canada.

E-mail: ashok.kakkar@mcgill.ca; Fax:+514-398-3797; Tel: 514-398-6912

${ }^{b}$ Department of Pharmacology and Therapeutics, McGill University 3655 Promenade Sir-William-Osler, Montreal, Quebec, H3G 1 Y6, Canada.E-mail: Dusica.maysinger@mcgill.ca;

Fax: + 514-398-6690; Tel: + 514-398-1264

$\dagger$ Electronic supplementary information (ESI) available: Details of synthesis and characterization of the dendrimers as well as the materials and methods related to the biology experiments are provided. See DOI: $10.1039 / \mathrm{c} 1 \mathrm{cc} 15354 \mathrm{~d}$
}

DA reactions has only been rarely exploited for drug delivery purposes, ${ }^{8}$ and to our knowledge, has not been demonstrated in the field of dendrimers. Release of conjugated therapeutic agents from well-defined and monodisperse scaffolds, carried out under a physiological range of temperatures, can offer a tremendous advancement in drug delivery. We report herein, a divergent synthesis of dendrimers on a four-arm flexible core, via a sequence of convenient CuAAC and DA "click" reactions. The periphery of such a dendrimer was subsequently conjugated to multiple units of lipoic acid (LA) using orthogonal Diels-Alder "click" chemistry. Lipoic acid (1,2-dithiolane3-pentanoic acid) is a therapeutic agent introduced into clinics as an antioxidant, chelating agent and transcription factor regulator. ${ }^{9}$ LA can facilitate re-establishing redox homeostasis in insulted cells by regulating intracellular antioxidants including vitamins $\mathrm{C}$ and $\mathrm{D}$, glutathione and redox responsive transcription factors. Dihydrolipoic acid is involved in the reduction of cystine to cysteine thereby facilitating the uptake of cysteine making it available for glutathione synthesis in cellular adaptation to oxidative stress. The effectiveness of LA to rescue neural cells was demonstrated in the primary cortical cultures exposed to hydrogen peroxide $\left(\mathrm{H}_{2} \mathrm{O}_{2}\right)$ and to 4-hydroxy2-nonenal (HNE)-mediated oxidative stress. LA in this study was used as a model therapeutic agent to demonstrate that it can be efficiently "clicked" to dendrimers, and that it can be released from them via retro-Diels-Alder reaction (rDA) at $37-42{ }^{\circ} \mathrm{C}$ (physiological/pathological range). A detailed investigation of these nanoconjugates and the released LA, revealed that they are noncytotoxic, and protect microglial cells from oxidative stress.

Synthesis of the dendrimers was carried out using building blocks compatible with CuAAc and DA "click" reactions, to be performed in sequence. In this regard, two different types of bifunctional units containing (i) an azide and two furan rings $\left(\mathbf{A z F u} \mathbf{u}_{\mathbf{2}}\right)$, and (ii) a maleimide and two acetylene moieties $\left(\mathbf{M a A c} \mathbf{c}_{\mathbf{2}}\right)$ were prepared. ${ }^{6}$ To synthesize the first generation dendrimer (G1-Fus, Scheme 1), $\mathbf{A z F u} \mathbf{u}_{2}$ was reacted via the $\mathrm{CuAAC}$ reaction with a core bearing four acetylene arms $\left(\mathbf{G 0 A c} \mathbf{c}_{4}\right)$, at room temperature for $2 \mathrm{~h}(80 \%$ yield $)$, or in a microwave reactor $\left(65{ }^{\circ} \mathrm{C}\right)$ for $30 \mathrm{~min}(64 \%$ yield $)$. The synthesis of the second generation dendrimer $\left(\mathbf{G} \mathbf{2}-\mathbf{A c}_{\mathbf{1 6}}\right)$, was

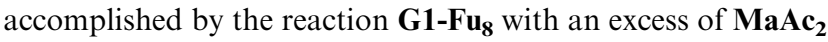
( $88 \%$ yield). Depending on the procedure used to perform 


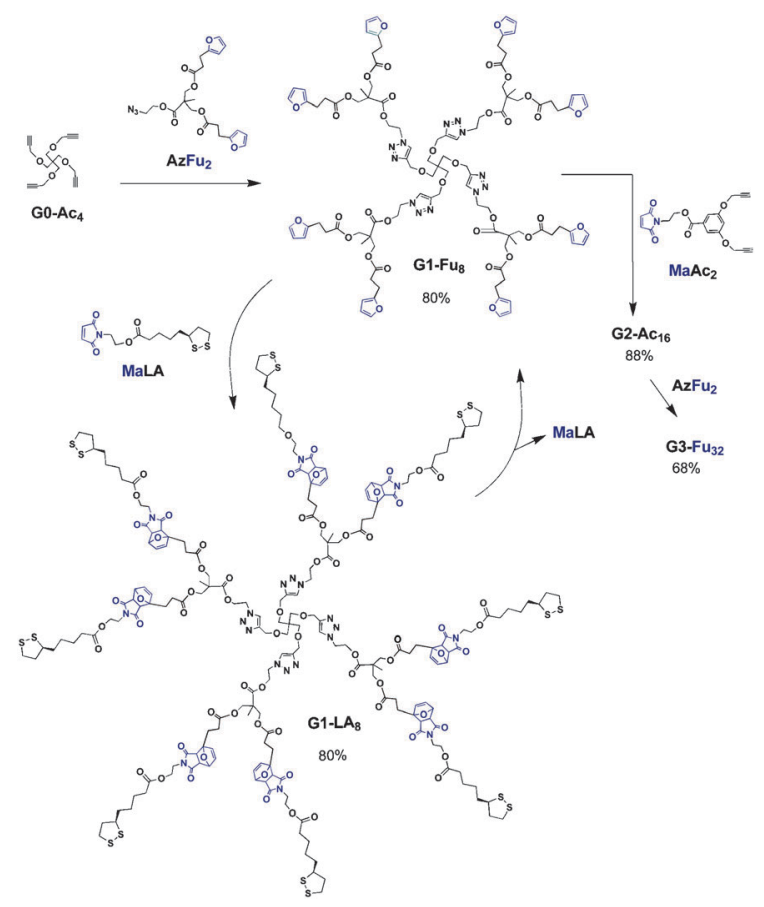

Scheme 1 Synthesis of the first generation dendrimer, G1-Fu, its conjugation with LA to give G1-LA $\mathbf{A}_{\mathbf{8}}$, and the preparation of the

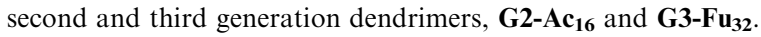

the reaction, different isomer ratios (endo/exo) for the DA cycloaddition were obtained. As expected, the more thermodynamically stable exo isomer was obtained as the major one $(90 \%)$ when the mixture was heated at $55{ }^{\circ} \mathrm{C}$ in ethyl acetate for 3 days. It is interesting to note that, when the reaction was performed using this strategy, $<5 \%$ of the peripheral furan moieties did not react with $\mathbf{M a A c} \mathbf{c}_{2}$, and a subsequent one day room temperature reaction was necessary to obtain a "defect free" dendrimer. The more kinetically stable endo isomer was obtained in a higher proportion $(50 \%)$ when the reaction was only performed at room temperature. However, 6-11 days were required for this reaction to go to completion. The reaction of $\mathbf{G} \mathbf{2}-\mathbf{A c}_{\mathbf{1 6}}$ with $\mathbf{A z F u} \mathbf{u}_{\mathbf{2}}$, via a CuAAC reaction, led to the synthesis of the third generation dendrimer, $\mathbf{G 3}-\mathbf{F} \mathbf{u}_{32}$ (68\% yield). We were intrigued to note that both the second and third generation dendrimers $\left(\mathbf{G} 2-\mathbf{A c} \mathbf{c}_{\mathbf{1 6}}\right.$ and $\left.\mathbf{G} 3-\mathbf{F u} \mathbf{u}_{\mathbf{3 2}}\right)$ underwent rDA disassembly at ambient temperature, to a larger extent with an increase in temperature, and this behavior prompted us to explore our dendritic system for drug-delivery purposes.

In order to conjugate LA molecules to the first generation dendrimer G1-Fu, $\mathbf{u}_{\mathbf{8}}$, we designed a building block containing both a maleimide and a LA unit (MaLA, Scheme 1), that could be "clicked" via DA reaction onto the dendrimer peripheral furan moieties. The synthesis of the LA conjugated

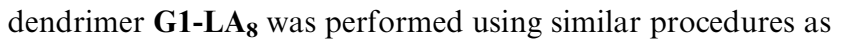

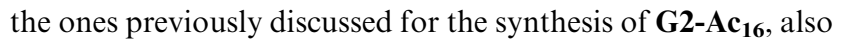
leading to similar endo/exo DA isomer ratios. We noted that G1-LA 8 also undergoes rDA disassembly, with a rate depending on the temperature, until the reaction equilibrium is reached. The time dependence of the MaLA release from the dendrimer was evaluated by ${ }^{1} \mathrm{H}$ NMR. Under the conditions used for the

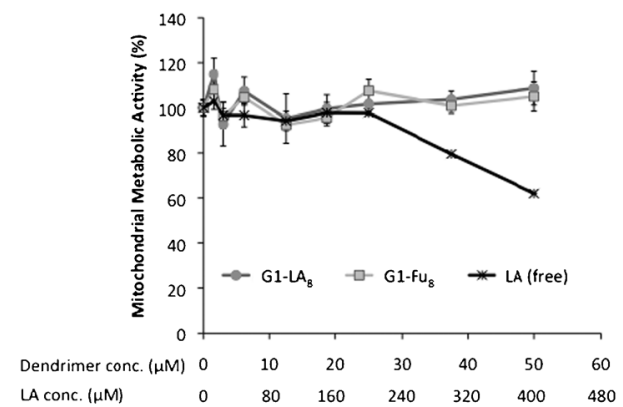

Fig. 1 Mitochondrial metabolic activity determined by MTT assay, upon exposure to $\mathbf{G 1 - L A _ { 8 }}\left(0-50 \mu \mathrm{M}\right.$, dendrimer conc.), $\mathbf{G 1 - F u _ { 8 }}$ $(0-50 \mu \mathrm{M}$, dendrimer conc.) or LA $(0-400 \mu \mathrm{M}$, LA conc.) for $24 \mathrm{~h}$. Note that LA is added in equimolar concentration to that of G1-LA. Metabolic activity (\%) in treated cells was expressed relative to controls (untreated cells that were set to $100 \%)(n=9)$.

experiment, it was found that the equilibrium is reached after approximately three days (MaLA release of 10, 40 and $50 \%$ at 22, 37 and $42{ }^{\circ} \mathrm{C}$, respectively), and that at physiological/ pathological temperatures $\left(37-42{ }^{\circ} \mathrm{C}\right), 20-30 \%$ of the MaLA units are released within the first $24 \mathrm{~h}$.

In order to demonstrate that LA retains its biological activity when provided as G1-LA 8 and to show that the dendrimer moieties are not cytotoxic, a series of experiments were carried out using living cells, i.e. microglia. This cell type was selected because microglia respond to even minute disturbances in the central nervous system; they efficiently patrol, survey and sense small disturbances. ${ }^{10}$ Depending on the extent of their activation, microglia can protect the neurons, or, if excessively active, they can contribute to their cell death.

The first aim was to show that microglia remain viable and functional upon exposure to dendrimer without and with LA. Non-treated cells served as negative controls and cells treated with unconjugated LA were positive controls in mitochondrial metabolic activity assay (Fig. 1). Microglia were exposed to a large concentration range of dendrimers $(0.005-50 \mu \mathrm{M})$ and mitochondrial metabolic activity/viability was assessed after $24 \mathrm{~h}$. Dendrimers were non-toxic during 24-48 h within the tested concentration range.

G1-LA $\mathbf{8}$ was then tested in cells exposed to three different insults: paraquat (1,1'-dimethyl-4,4'-bipyridinium dichloride), $\mathrm{H}_{2} \mathrm{O}_{2}$ and lipopolysaccharide (LPS). It was anticipated that LA released from the dendrimer would retain its biological activity and would be capable of protecting the cells from reactive oxygen species (ROS), particularly from an excess of superoxide $\left(\mathrm{O}_{2}{ }^{-}\right)$induced by paraquat (PQ) (Fig. 2A and B), $\mathrm{H}_{2} \mathrm{O}_{2}$ (Fig. 2C and D) or nitric oxide generated in microglia treated with LPS (Fig. S3, see ESI). PQ is a widely used herbicide causing damage to multiple organs (liver, kidney, heart, lungs, central nervous system). ${ }^{11}$ It is commonly used to produce injury to dopaminergic neurons mimicking certain features of Parkinson's disease in experimental animals. In order to quantify the superoxide level within the cells upon treatment with PQ, dihydroethidium (DHE) was used. DHE oxidation by superoxide generates ethidium, whose fluorescence can be easily monitored. ${ }^{12}$ Interestingly, the treatment of PQ-stressed microglia cells with G1-LAs, or its precursor $\mathbf{G 1 - F \mathbf { u } _ { 8 }}$, resulted in a significant reduction of intracellular 


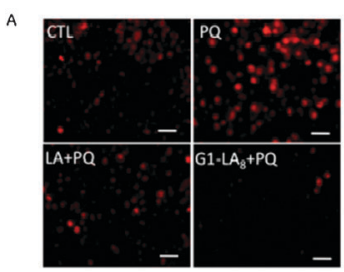

B

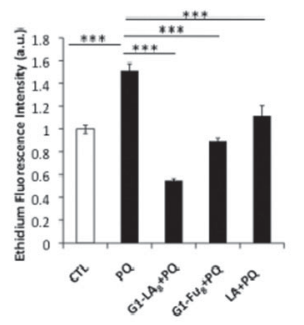

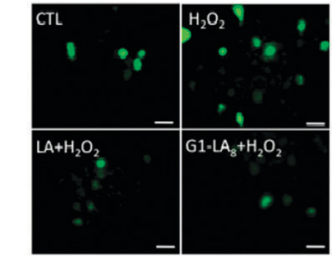

D

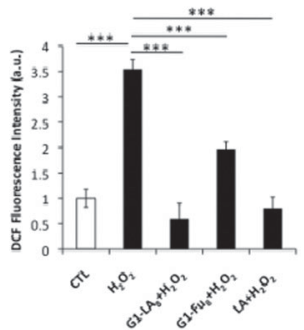

Fig. 2 Generation of superoxide and reactive oxygen species (ROS) by paraquat (PQ) and $\mathrm{H}_{2} \mathrm{O}_{2}$. Microglia were treated with G1-LA $(12.5 \mu \mathrm{M}), \mathbf{G 1}-\mathbf{F u}_{\mathbf{8}}(12.5 \mu \mathrm{M})$ or $\mathrm{LA}$ in equimolar concentration $(100 \mu \mathrm{M})$ for $24 \mathrm{~h}$. (A) Fluorescent micrographs showing superoxide anion $\left(\mathrm{O}_{2}{ }^{-}\right)$generation following PQ exposure $(500 \mu \mathrm{M}, 3 \mathrm{~h})$ using dihydroethidium (DHE). (B) Spectrofluorometric determination of ethidium fluorescence intensity (arb. units). Controls (CTL) $=$ untreated cells $(n=6)$. (C) Fluorescent micrographs showing ROS generation following $\mathrm{H}_{2} \mathrm{O}_{2}$ exposure $(200 \mu \mathrm{M}, 3 \mathrm{~h})$ using DCFH-DA; Scale bar, $20 \mu \mathrm{m}$. (D) Spectrofluorometric determination of DCF fluorescence intensity (arbitrary units relative to control set at 1).

superoxide (Fig. 2A and B), to an even larger extent than with initially unbound LA. The ratio between the cells labelled with ethidium (red) and the total number of cells corroborated the results obtained by spectrofluorometric superoxide determinations (Fig. 2A and B).

When microglial cells were exposed to $\mathrm{H}_{2} \mathrm{O}_{2}$, a common inducer of oxidative stress (Fig. 2C and D), a general ROS marker $2^{\prime}, 7^{\prime}$-dichlorodihydrofluorescein diacetate (DCFH-DA) was used. Intracellular DCFH-DA reacts with different ROS species yielding a highly fluorescent compound, 2', $7^{\prime}$-dichlorofluorescein (DCF). The fluorescent signal was significantly reduced in cells treated with dendrimer, coupled with LA or devoid of LA. The observation that $\mathbf{G 1 - F \mathbf { u } _ { 8 }}$ can reduce ROS (although not as effectively as a control agent LA), merits further investigations to explain the mechanisms involved in ROS suppression in microglia and other cell types.

We next examined the protective effect of dendrimer with and without LA in cells exposed to LPS, a well established endotoxin released from Gram negative bacteria, which is commonly used to mimic bacterial infection and to activate microglia. The results (Fig. S3, see ESI $\dagger$ ) clearly show that LPS induced an increase in nitric oxide which was significantly reduced, with LA, and the dendrimer with or without bound LA (i.e. G1-Fus and G1-LA, $\mathbf{u}_{\mathbf{8}}$ ). We are intrigued by the activity

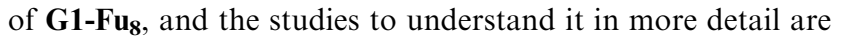
currently being pursued in our laboratories.
In summary, our studies demonstrate that the functionalization of dendrimers using Diels-Alder reaction, at ambient temperature, is a suitable strategy to prepare drug conjugates carrying multiple drug molecules which can be released under physiological $\left(37^{\circ} \mathrm{C}\right)$ or pathological $\left(42^{\circ} \mathrm{C}\right)$ conditions. The release of an active and powerful anti-inflammatory and antibacterial agent by rDA could be particularly attractive for therapeutic interventions against bacterial infections accompanied by an increased local or systemic body temperature. The fine-tuning of $\mathrm{rDA}$ reactions within the physiologically and pathologically relevant temperature range makes the synthesis of other drugdendrimer conjugates widely applicable, and offers advantages over free drugs by providing better temporal and spatial drug concentration at the sites of excessive ROS production and inflammation.

We would like to thank Natural Sciences and Engineering Research Council of Canada, (NSERC), Canadian Institutes of Health Research (CIHR) and Fonds de Recherche en Santé du Québec (FRSQ) and Centre for Self-Assembled Chemical Structures (Fonds québécois de la Recherche sur la Nature et les Technologies) for financial support.

\section{Notes and references}

1 P. T. Hammond, ACS Nano, 2011, 5, 681; O. C. Farokhzad and R. Langer, ACS Nano, 2009, 3, 16.

2 R. Roy, J. N. Cambre and B. S. Sumerlin, Prog. Polym. Sci., 2010, 35, 178; L. Zha, B. Banik and F. Alexis, Soft Matter, 2011, 7, 5908; G. M. Soliman, A. Sharma, D. Maysinger and A. Kakkar, Chem. Commun., 2011, 47, 9572.

3 Y. Pang, J. Liu, Y. Su, J. Wu, L. Zhu, X. Zhu, D. Yan and B. Zhu, Polym. Chem., 2011, 2, 1661.

4 U. Boas, J. B. Christensen and P. M. H. Heegaard, Dendrimers in Medicine and Biotechnology, New Molecular Tools, RSC Publishing, Thomas Graham House, Cambridge, 2006; R. Hourani and A. Kakkar, Macromol. Rapid Commun., 2010, 31, 947; M. A. Mintzer and M. W. Grinstaff, Chem. Soc. Rev., 2011, 40, 173.

5 G. Franc and A. K. Kakkar, Chem. Commun., 2008, 5267; G. Franc and A. K. Kakkar, Chem. Soc. Rev., 2010, 39, 1536; A. Sanyal, Macromol. Chem. Phys., 2010, 211, 1417.

6 P. Antoni, M. J. Robb, L. Campos, M. Montanez, A. Hult, E. Malmström, M. Malkoch and C. J. Hawker, Macromolecules, 2010, 43, 6625; A. Vieyres, T. Lam, R. Gillet, G. Franc, A. Castonguay and A. K. Kakkar, Chem. Commun., 2010, 46, 1875 .

7 M. Gingras, J.-M. Raimundo and Y. M. Chabre, Angew. Chem., Int. Ed., 2007, 45, 1010; E. R. Gillies and J. M. J. Fréchet, Bioconjugate Chem., 2005, 16, 361; J. R. McElhanon and D. R. Wheeler, Org. Lett., 2001, 3, 2681; M. M. Kose, G. Yesilbag and A. Sanyal, Org. Lett., 2008, 2353.

8 T. Dispinar, R. Sanyal and A. Sanyal, J. Polym. Sci., Part A: Polym. Chem., 2007, 45, 4545.

9 L. Packer and E. Cadenas, J. Clin. Biochem. Nutr., 2011, 48, 26.

10 M. B. Graeber, Science, 2010, 330, 783.

11 T. Fukushima, K. Tanaka, H. Lim and M. Moriyama, Environmental Health and Preventive Medicine, 2002, 7, 89; J. M. Morán, M. A. Ortiz-Ortiz, L. M. Ruiz-Mesa and J. M. Fuentes, J. Biochem. Mol. Toxicol., 2010, 24, 402.

12 J. Lovrić, S. J. Cho, F. M. Winnik and D. Maysinger, Chem. Biol., 2005, 12, 1227; F. R. Laurindo, D. C. Fernandes and C. X. Santos, Methods Enzymol., 2008, 441, 237. 